\title{
Dynamic Decomposition Analysis and Forecasting of Energy Consumption in Shanxi Province Based on VAR and GM $(1,1)$ Models
}

\author{
Herui Cui $(\mathbb{D}$, Ruirui Wu $(\mathbb{D}$, and Tian Zhao \\ Department of Economics and Management, North China Electric Power University, Baoding 071003, China \\ Correspondence should be addressed to Ruirui Wu; wrr20180131@163.com
}

Received 18 May 2018; Accepted 7 June 2018; Published 2 July 2018

Academic Editor: Vincenzo Bianco

Copyright (C) 2018 Herui Cui et al. This is an open access article distributed under the Creative Commons Attribution License, which permits unrestricted use, distribution, and reproduction in any medium, provided the original work is properly cited.

\begin{abstract}
Environmental issues caused by energy consumption have attracted increasing attention recently. Shanxi Province, a typical energy-dominated region in China, has long-term dependency on coal industry generating extensive economic growth, which is detrimental to green development. Distinguished from previous studies ignoring driving factors of energy consumption, this paper establishes a vector autoregression (VAR) model to dynamically identify the drivers of energy consumption based on STIRPAT model in Shanxi Province from 1990 to 2015. It can be obtained from the impulse response analysis that a positive shock in population, GDP, and urbanization level, respectively, positively affect energy consumption, and a positive change in technology negatively affects energy consumption in the long run. The variance decomposition results indicate that fluctuation in energy consumption explained by the innovation of the urbanization level accounts for $23.18 \%$, which plays a prevailing role in increasing energy consumption. Meanwhile, the forecasting results of GM $(1,1)$ model manifest that energy consumption in Shanxi Province generally has an increasing trend from 2016 to 2025. Consequently, Shanxi can achieve green development through optimizing energy structure, promoting the transformation of resource-based cities, and promoting low-carbon technological innovation. This paper can be available for other resource-based regions analogous to Shanxi.
\end{abstract}

\section{Introduction}

As the energy is the significant resource and motive power, its cheap and stable supply is the safeguard of the economic and social development [1]. With China's enormously economic growth, energy consumption shows a rapid increase trend [2-4]. However, the combustion of fossil energy is the main source of $\mathrm{CO}_{2}$ emissions, which makes China's government face great challenges of global climate change and domestic environment protection to guarantee energy supply and energy-related environment security [5]. Meanwhile, China has now become the largest $\mathrm{CO}_{2}$ emitter in the world and joined "The Paris Agreement" to work together for coping with "greenhouse effect" globally after 2020 [6]. This implies that it is essential for the decision-makers to implement appropriate energy policy for China's sustainable development.

As a spatially large developing country, China not only has prominent regional differences in climate and geography, but also has great diversities in energy endowments, economic development patterns, and levels of household consumption. It is critical to identify the main driving forces of energy consumption growth at the regional level, so that appropriate regional-specific energy conservation policies can be adopted.

Shanxi Province in central China is rich in mineral resources, among which coal, coalbed methane (CBM), bauxite, and iron are the most affluent. Specifically, coal has reserves of $2767.85 \times 10^{8}$ tons, accounting for $20.1 \%$ of the country's total reserves. And the reserves of $\mathrm{CBM}$ are $1825.16 \times 10^{8} \mathrm{~m}^{3}$, accounting for $88.2 \%$ of the total. Shanxi Province is a traditional coalmining region and a significant energy and industrial base in China. Since 1949, Shanxi has yielded 15.3 billion tons of coal which account for one-fourth of the national total [7], with the net amount of coal transferred being 10.4 billion tons accounting for three quarters of the national total. And coke production and the amount of 
the transfer account for $40 \%$ and $60 \%$ of the national total, respectively [8], making remarkable contribution towards China's economic development.

However, the long-term extensive economic growth model in Shanxi has engendered excessive dependence on the coal and a single industrial structure, inducing the issues of resource exhaustion and the formation of many subsidence and goaf areas. Meanwhile, the primary energy consumed in Shanxi was produced by the burning of coal, which accounts for a large share of greenhouse gas (GHG) emissions. Moreover, energy demand continues to increase and so does the air pollution, causing severe health problems, crop damage, and acid rain, all of which are taking a social and economic toll. Hence, it is beneficial and necessary to study the energy consumption in Shanxi Province.

Literature based on energy consumption in China can be generally classified as national and provincial angles. Many scholars have researched energy consumption in China from a nationwide perspective, and the initial study focused on the dual relationship between energy consumption and economic growth. Soytas and Sari [9] examined relationship between energy consumption and economic development in China and found that there was no causal relationship between total energy consumption and GDP. Talha and Cakar [10] researched the causal relationship between energy consumption and real GDP based on asymptotic distribution theory. As for the driving factors of energy consumption, Zhang and Song [11] used Logarithmic Mean Divisia Index (LMDI) method to study the factors affecting the changes in energy consumption in China and uncovering the economic growth effect was the primary factor. Li et al. [12] identified the factors driving China's energy consumption and demonstrated demand elasticity of impact factors changed regularly. Xie et al. [13] studied autocorrelation and influenced factors of energy consumption change in China based on the spatial autocorrelation analysis and the autoregression model. There are also some studies aimed at energy consumption predictions. Pao et al. [14] forecasted carbon emissions, energy consumption in China based grey model. Xiong et al. [15] proposed a novel GM $(1,1)$ model to predict China's energy consumption and production. Wang et al. [16] estimated primary energy consumption based on the hybrid AMVO-SVM model to predict the energy consumption of China.

Literature concerning the energy consumption at national level gave macro conclusions which were not applicable in all regions with a noticeable diversity across every region throughout China. Thus, scholars continued to study more concretely several representative provinces and cities. Most of the studies mainly analyzed the energy consumption of economically developed regions from a static point of view.

Wang and Feng [17] discussed energy resource, production, and consumption in Jiangsu province and put forward a long-term policy to ensure the sustainable development. Cai and Jiang [18] explored differences in energy consumption between rural and urban households and designed relative conservation implications in Shaanxi Province. Ren et al. [19] studied energy consumption and carbon emissions during the urbanization of Shandong Province and demonstrated urbanization had distinct positive impacts on energy consumption and carbon emissions. Chong et al. [20] analyzed the driving factors of energy consumption growth in Guangdong Province based on the LMDI decomposition model. Feng et al. [21] used system dynamics approach to probe into the influence of driving factors selected on the energy consumption and $\mathrm{CO}_{2}$ emission trends in Beijing. There are also a few studies that have comprehensively analyzed the energy consumption of Chinese provinces. Zhang and Lahr [22] applied the structural decomposition method to uncover the regional disparities in energy consumption and proposed regional-specific policies. Wang et al. [23] investigated the relationship between urbanization, energy consumption, and $\mathrm{CO}_{2}$ emissions based on the data from 30 Chinese provinces by a panel data model.

In terms of Shanxi Province, Wang [24] et al. conducted an empirical analysis of the relationship between economic growth and energy supply and demand in Shanxi Province. The results showed that energy consumption and energy supply together constituted a unidirectional driving engine for economic growth in Shanxi Province. Xu et al. [25] used the SVAR model to analyze the dynamic relationship between urbanization and energy consumption and concluded that urbanization in the medium and long term contributed more to the variance in energy consumption. Li and Yang [26] analyzed the energy consumption system and related subsystems in Shanxi Province by constructing a system dynamics model and provided corresponding policy recommendations to ensure sustainable development. Zhai [27] used the ARIMA model to predict the time series of energy consumption in Shanxi Province.

Shanxi is a national resource-based economy restructuring comprehensive reform pilot area in China. Reviewing existing literature, however, research conducted on driving energy consumption in Shanxi has been very scanty. Moreover, previous studies have mainly emphasized on the relationship between energy consumption and a few concerning variables but ignored its driving factors. Meanwhile, the ARIMA model frequently adopted does not have high prediction accuracy when the sample size is small.

In summary, to make a contribution to filling the gaps in existing studies, we conduct the paper from the following aspects. First, we use the Stochastic Impacts by Regression on Population, Affluence and Technology (STIRPAT) model to explore determinants of energy consumption of Shanxi Province at the regional level. Second, considering the dynamical research of energy systems lacked in Shanxi, a vector autoregression (VAR) model based STIRPAT model is established to quantitatively analyze the influencing factors of energy consumption growth, which is not subject to traditional economic theory and to avoid the estimated error caused by multicollinearity [26]. Third, with relatively little data, the grey forecasting model provides approximately realistic forecast values of energy consumption for corresponding policy establishment [28]. Finally, conclusions and policy recommendations to boost green sustainable development in Shanxi are presented based on results received, which is essential for controlling energy consumption and deserves 
further study for other resource-based regions analogous to Shanxi.

Therefore, this paper sheds light on the current studies in the following points. (1) It is the first try to combine the VAR model and the extended STIRPAT model to analyze the energy consumption issue in Shanxi Province, a vital energy-dominated region in China. (2) The influenced factors selected in the extended STIRPAT model are distinguished from previous research, to explore a different study perspective concerning energy consumption in Shanxi. (3) It is the first time that we use the GM $(1,1)$ method, with relatively little data, to forecast energy consumption in Shanxi from 2016 to 2025.

\section{Method}

2.1. VAR Model. The VAR model, one of the easiest models to analyze and predict multiple related economic indicators, is proposed by Sims [29] based on statistic characteristic of data comprehensively making use of multiple variables information. Further, a VAR model can better cover enough information to simulate the system than that using merely one single time series, providing more realistic predicted values in forecasting when making the near future prediction.

The multivariable VAR (P) model can be obtained by

$$
y_{t}=\Gamma_{0}+\Phi_{1} y_{t-1}+\ldots+\Phi_{p} y_{t-p}+\varepsilon_{t}, \quad t=1, \ldots, T
$$

where $y_{t}$ is a $k \times 1$ vector of endogenous variables, $\Phi_{t} k \times k$ coefficient matrices, $\varepsilon_{t}$ the one-step ahead prediction error with variance-covariance matrix $\Sigma, \Gamma_{0}$ the intercept, and $p$ the lag length. As for the VAR model, the single coefficient can only reflect a local relationship and fails to capture the overall impacts of one variable on the other, which is meaningless to study the coefficients in this model. Thus, based on the VAR model established, the main purpose on this occasion is to analyze the dynamic relations between variables by impulse response analysis and variance decomposition.

Impulse response analysis can comprehensively reflect not only the dynamic relationship among various variables but also influence of the change in an endogenous variable on others in the system. Specifically, it demonstrates impacts on the current or future value when a random error term is shocked from inner or outer system.

Equation (1) corresponds with the stability condition if roots of the determinant are outside the unit circle, so that in this case the VAR model can be shown as an infinite vector VMA process, and (1) can be rewritten by

$$
y_{t}=\Theta(L) \varepsilon_{t}
$$

where $L$ is the lag operator. For two conditioning random variables, if $y_{1}$ is given a unit of impulse in the base period: $\varepsilon_{1 t}=1, t=0 . \varepsilon_{1 t}=0, t=$ others, $\varepsilon_{2 t}=0, t=0,1,2, \ldots$, the impulse response function of $y_{2}$ caused by $y_{1}$ can be expressed as

$$
\begin{aligned}
& t=0, \quad y_{20}=\theta_{21}^{(0)} \\
& t=1, \quad y_{21}=\theta_{21}^{(1)} \\
& t=2, \quad y_{22}=\theta_{21}^{(2)}, \ldots
\end{aligned}
$$

Variance decomposition will decompose the variance of one variable in the system into each disturbance, to analyze the innovation of the endogenous variables from the impact of each structure, so that the significance of various structural shocks can be further explored. In this case, (2) can be rewritten as

$$
\begin{aligned}
y_{i t}=\sum_{j-1}^{k}\left(\theta_{i j}^{(0)} \varepsilon_{j t}+\theta_{i j}^{(1)} \varepsilon_{j t-1}\right. & \left.+\theta_{i j}^{(2)} \varepsilon_{j t-2}+\ldots\right), \\
& \quad i=1,2, \ldots, k, t=1,2, \ldots, T
\end{aligned}
$$

The content of each bracket denotes the sum of influence of the disturbance $\varepsilon_{j}$ from the infinite past to the present, which can be evaluated by the variance. Supposing $\varepsilon_{j}$ is no serial correlation, variances of $y_{i}$ can be decomposed into $\mathrm{k}$ types of unrelated effects. Hence, the relative variance contribution rate is defined to determine how much the variance of disturbance contributes to the variance of $y_{i}$.

If the VAR model satisfies the stability condition, the approximate relative variance contribution rate is shown as follows:

$$
R V C_{j \rightarrow i}(s)=\frac{\sum_{q=0}^{s-1}\left(\theta_{i j}^{q}\right)^{2} \sigma_{j j}}{\sum_{j=1}^{k}\left\{\sum_{q=0}^{s-1}\left(\theta_{i j}^{(q)}\right)^{2} \sigma_{j j}\right\}}
$$

where $\sigma_{j j}$ refers to standard deviation of $\varepsilon_{j}$. The greater the $\sigma_{j j}$, the larger the impact of the $j_{t h}$ variable on the $i_{t h}$ variable.

2.2. GM (1,1) Model. The grey system was firstly proposed by Deng [30]. The grey forecasting model can be applied in cases with relatively fewer statistics, which is suitable for predicting the competitive environment where decisionmakers can refer to mere limited historical data. The GM $(1,1)$ procedure can be summarized as follows.

Step 1. The nonnegative raw time-sequence data $X^{(0)}$ is defined as

$$
\begin{aligned}
X^{(0)}=\left\{X^{(0)}(1), X^{(0)}(2), \ldots, X^{(0)}(n)\right\} & \\
X^{(0)}(k) & >0, k=1,2, \ldots, n .
\end{aligned}
$$

Step 2. Take accumulated generating operation (AGO) on $X^{(0)}$ into a new sequence $X^{(1)}$ :

$$
X^{(1)}=\left\{X^{(1)}(1), X^{(1)}(2), \ldots, X^{(1)}(n)\right\}
$$

where $X^{(1)}(k)=\sum_{i=1}^{k} X^{(0)}(i), k=1,2, \ldots, n$. 
Step 3. The basic form of $\operatorname{GM}(1,1)$ model is obtained by the following equation:

$$
\frac{d X^{(1)}}{d t}+a X^{(1)}=b
$$

where $a$ is a developing coefficient and $b$ represents the grey input. Parameter $\hat{a}$ is represented by

$$
\hat{a}=\left(B^{T} B\right)^{-1} B^{T} Y
$$

where

$$
B=\left[\begin{array}{cccc}
-\frac{1}{2}\left(X^{(1)}(1)+X^{(1)}(2)\right) & 1 & \cdots & 1 \\
-\frac{1}{2}\left(X^{(1)}(2)+X^{(1)}(3)\right) & 1 & \cdots & 1 \\
\vdots & & \vdots & \\
-\frac{1}{2}\left(X^{(1)}(n-1)+X^{(1)}(n)\right) & 1 & \cdots & 1
\end{array}\right],
$$

and $Y_{n}=\left[X_{1}^{(0)}(2), X_{1}^{(0)}(3), \cdots, X_{1}^{(0)}(n)\right]^{T}$.

Step 4. According to the estimated coefficients $a$ and $b$, the response equation and the prediction value can be obtained by

$$
\begin{aligned}
& \stackrel{\wedge}{X}^{(1)}(k+1)=\left(X^{(1)}(0)-\frac{b}{a}\right) e^{-a k}+\frac{b}{a} \\
& \stackrel{\wedge}{X}^{(0)}(k+1)=\stackrel{\wedge}{X}^{(1)}(k+1)-\stackrel{\wedge}{X}^{(1)}(k), \quad \\
& \quad k=1,2, \ldots, n .
\end{aligned}
$$

To demonstrate the reliability of the GM $(1,1)$ model, the posterior variance test is utilized to discuss the results of fitting and prediction. Criteria of the posterior variance test contain the indicators, posterior variance ratio $C$, and small error probability $P$. The posterior variance ratio $C$ is defined as

$$
C=\frac{s_{2}}{s_{1}}
$$

where $s_{1}{ }^{2}=(1 / n) \sum_{k=1}^{n}\left(X^{(0)}(k)-\bar{X}^{(0)}(k)\right)^{2}$ and $s_{2}{ }^{2}=$ $(1 / n) \sum_{k=1}^{n}\left(\varepsilon^{(0)}(k)-\bar{\varepsilon}^{(0)}(k)\right)^{2}, k=1,2, \ldots, n . \varepsilon^{(0)}(k)$ represents the residual between the $\hat{\Lambda}^{(0)}(k)$ and $X^{(0)}(k)$

The small error probability is defined as

$$
P=\left\{\left|\varepsilon^{(0)}(k)-\bar{\varepsilon}^{(0)}(k)\right|<0.6745 s_{1}\right\}
$$

The range of indicator values $C$ and $P$ is such that $C>0$, $0 \leq P \leq 1$, where $P>0.95$ and $C<0.35$ indicate a highly accurate forecast, $P>0.60$ and $C<0.80$ indicate a reasonable forecast, and $P<0.60$ and $C>0.80$ indicate an unreasonable range
2.3. STIRPAT Model. To study the driving factors of the environment, Ehrlich [31] first proposed IPAT model in 1971, i.e.,

$$
I=P A T
$$

where $I$ denotes the environment pressure, $P$ represents population, $A$ indicates affluence, and $T$ is technology. The IPAT equation hypothesized that environmental impact changes proportionally or monotonically, which is not accordance with the reality condition.

To overcome this limitation, Rosa and Dietz [32] developed a model named STIRPAT. The specific model can be described as

$$
I=a P^{b} A^{c} T^{d} e
$$

where $I, P, A$, and $T$ are the same variables as those in (14); $a$, $b, c$, and $d$ are coefficients representing stochastic impacts; $e$ denotes error term. The natural logarithm form is shown in

$$
\ln (I)=\ln (a)+b \ln (P)+c \ln (A)+d \ln (T)+\ln (e)
$$

The STIRPAT model is entitled to be extended by incorporating extra factors for researching more possible causes for environmental pressure [33]. Therefore, how to select influenced factors is the key point of the whole study. In order to investigate the impacts of the driving forces of the energy consumption in Shanxi Province, considering the specific development situation and learning from relevant research experience, we rewrote (16) as follows:

$$
\begin{aligned}
\ln (I)= & \ln (a)+\beta_{1} \ln (P)+\beta_{2} \ln (A)+\beta_{3} \ln (T) \\
& +\beta_{4} \ln (U)+\ln (e)
\end{aligned}
$$

Energy consumption can be regarded as a kind of environmental impact because it consumes natural resources, denoted by EC. $P$ denotes population size, $A$ is measured by per capita GDP, $T$ represents the total factor productivity (TFP), $U$ represents urbanization level, and $\beta_{1}, \beta_{2}, \beta_{3}$, and $\beta_{4}$ are estimation parameters. So we rewrote (17) as follows:

$$
\begin{aligned}
\ln (E C)= & \ln (a)+\beta_{1} \ln (P)+\beta_{2} \ln (G D P)+\beta_{3} \ln (T) \\
& +\beta_{4} \ln (U R B)+\ln (e)
\end{aligned}
$$

2.4. Data Illustration and Collection. The paper adopted the data interval 1990-2015; all data in this paper covering the period of 1990-2015 are derived from the Shanxi Statistical Yearbook [34]. More specifically, technology progress is measured by TFP, because this indicator accurately reflects the level of technology. This paper selects DEA-Malmquist productivity index method based on dynamic nonparametric frontier production surface. This method is based on balancing panel data containing multiple decision units, applying input-output data, identifying the best business decisionmaking unit for technical efficiency, and constructing the production frontier based on it. The technical efficiency level index is obtained by comparing the actual input or output value of the production process with the optimal value. And 
TABLE 1: The definitions of variables.

\begin{tabular}{lccc}
\hline Variable & Symbol & Definition & Unit \\
\hline Energy consumption & EC & Total energy consumption & $10^{4}$ tce \\
Population & $\mathrm{P}$ & Total population & $10^{4}$ units \\
Economic level & GDP & GDP per capital converted to constant prices of 1990 & Yuan \\
Technology & $\mathrm{T}$ & Total factor productivity & $\%$ \\
Urbanization level & URB & Proportion of city population over the total population & $\%$ \\
\hline
\end{tabular}

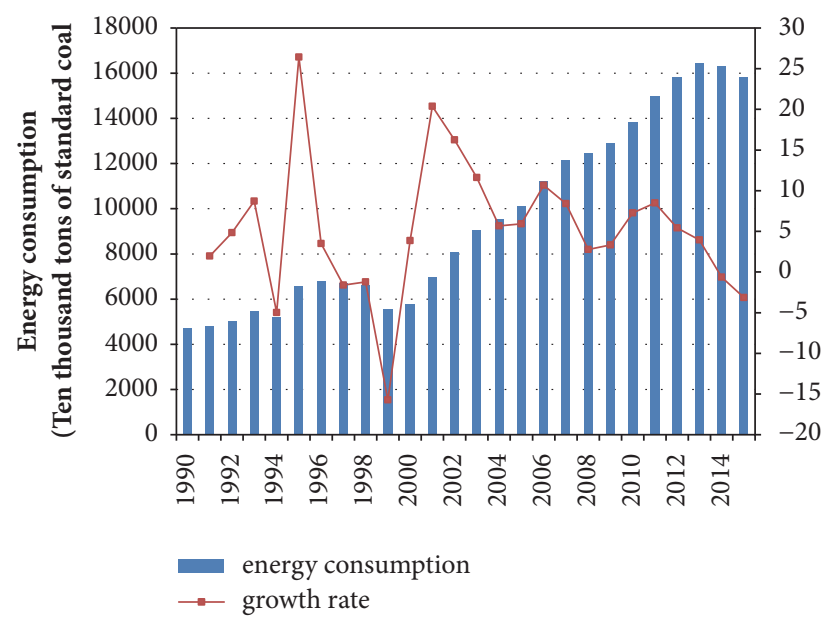

FIGURE 1: Change in energy consumption in Shanxi Province.

this method allows technology inefficiency to exist and is therefore widely used for total factor productivity estimation. The DEA 2.1 software is used in this paper to measure TFP of Shanxi Province in each year. The key of the DEA-Malmquist productivity index method is the selection of input and output variables. The inputs of this article are selected from the two indicators of physical capital and labor. The physical capital is represented by the capital stock, the labor index is selected by the number of employed people, and the output index is expressed by GDP. This paper draws on the research of Shan Haojie [35] to select capital stock data estimation methods and indicators. In summary, the definitions of all variables are shown in Table 1.

\section{Result}

3.1. The Changing Tendency of Energy Consumption. Figure 1 shows the trend of Shanxi's energy consumption during 1990 and 2015. We can obviously see that the total energy consumption changes by various stages. The first stage is from 1990 to 1999, during which energy consumption experienced a gentle growth with an average annual growth rate of $2.43 \%$. The second stage is from 2000 to 2013, during which energy consumption of Shanxi increased rapidly with an annual growth rate of $8.14 \%$. The third stage is from 2013 to 2015, when energy consumption of Shanxi suddenly decreased. This may be attributed to the reason that the downturn of coal markets and the rapid drop of energy prices since $2013 \mathrm{had}$

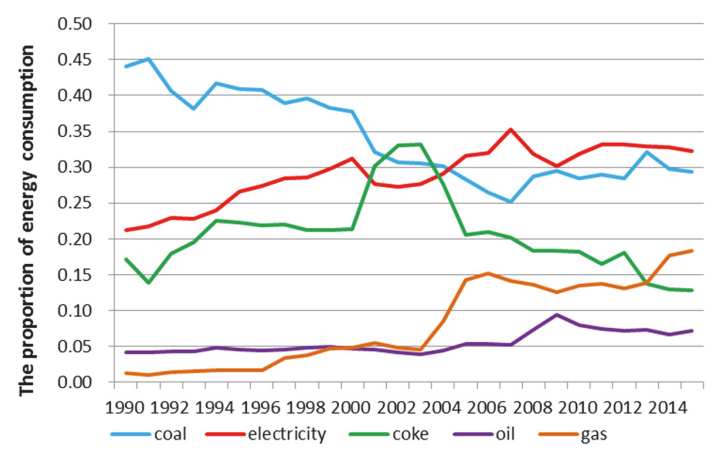

FIGURE 2: Changes in proportion of each source to energy consumption.

caused a severely negative shock to the economy of Shanxi Province.

As is shown in Figure 2, from sectional perspective, the coal, electricity, and coke remained to be leading parts in energy structure in the past, accounting for approximately $90 \%$ of total energy consumption from 1994 to 2004. While this percentage declined with small fluctuations from 2005 to 2015 , it has still remained over $80 \%$. As for the oil, its consumption has always accounted for a comparatively tiny proportion of the whole; only $7.2 \%$ of total energy consumption in 2015. Last but not least, although the percentage of natural gas showed a general trend of growth, the increasing rate was very slow. This indicates that relevant departments should vigorously develop clean energy and improve energy structure.

The original VAR model requires that each variable should be stationary. For nonstationary variables, the differential processing needs to be performed to obtain a stationary time series to establish a VAR model, which will nevertheless lose the corresponding level information. With the help of cointegration theory, VAR models can be directly established as long as there exist cointegration relationships between variables [36].

It should be the first to conduct the stationarity test to determine the stationarity of variables and its order of integration, with the test methods including the augmented Dickey-Fuller (ADF) test and the nonparametric PhillipsPerron (PP) test [37]. In this paper, the ADF test is employed.

From Table 2, as for the logarithm form of EC, P, GDP, T, and URB, the correlative ADF statistics are greater than the critical value at the $10 \%$ significance level, indicating that the logarithm forms of these variables are all nonstationary. Tests 
TABLE 2: Statistical results of ADF test.

\begin{tabular}{|c|c|c|c|c|c|c|}
\hline Variable & Test type $(\mathrm{c}, \mathrm{t}, \mathrm{p})$ & ADF statistics & $10 \%$ critical values & $5 \%$ critical values & $\mathrm{P}$ value & Result \\
\hline $\operatorname{Ln}(\mathrm{EC})$ & $(c, 1,9)$ & 0.8753 & -3.3103 & -3.7332 & 0.9993 & Nonstationary \\
\hline $\mathrm{D}(\operatorname{Ln}(\mathrm{EC}))$ & $(c, 0,0)$ & $-3.9381^{* * *}$ & -2.6336 & -2.9919 & 0.0063 & Stationary \\
\hline $\operatorname{Ln}(\mathrm{PGDP})$ & $(c, 1,1)$ & -2.1390 & -3.2431 & -3.6122 & 0.4997 & Nonstationary \\
\hline $\mathrm{D}(\operatorname{Ln}(\mathrm{PGDP}))$ & $(c, 0,0)$ & $-3.3258^{* *}$ & -2.6336 & -2.9919 & 0.0297 & Stationary \\
\hline $\operatorname{Ln}(\mathrm{P})$ & $(c, 1,0)$ & -2.6685 & -3.2381 & -3.6032 & 0.2565 & Nonstationary \\
\hline $\mathrm{D}(\operatorname{Ln}(\mathrm{P}))$ & $(c, 0,0)$ & $-5.0175^{* * *}$ & -2.6336 & -2.9919 & 0.0005 & Stationary \\
\hline $\operatorname{Ln}(\mathrm{T})$ & $(c, 1,0)$ & -3.2312 & -3.2381 & -3.6032 & 0.1012 & Nonstationary \\
\hline $\mathrm{D}(\operatorname{Ln}(\mathrm{T}))$ & $(c, 0,1)$ & $-5.0734^{* * *}$ & -2.6388 & -2.9981 & 0.0005 & Stationary \\
\hline $\operatorname{Ln}(\mathrm{URB})$ & $(c, 1,0)$ & -2.8535 & -3.2381 & -3.6032 & 0.1930 & Nonstationary \\
\hline $\mathrm{D}(\operatorname{Ln}(\mathrm{URB}))$ & $(c, 0,0)$ & $-6.9982^{* * *}$ & -2.6336 & -2.9919 & 0.0001 & Stationary \\
\hline
\end{tabular}

Note: $(c, t, P)$ is the test type, where $c$ is the intercept, $t$ is the trend, $p$ is the lag length, and Zero value means there is no such item. Lag order selected by the SIC criterion. $* * *, * *$, and $*$, respectively, denote the rejection of the null hypothesis at $1 \%, 5 \%$, and $10 \%$ significance level.

TABLE 3: Optimum lags selection.

\begin{tabular}{lccccc}
\hline Lag & LR & FPE & AIC & SC & HQ \\
\hline 0 & NA & $3.42 \mathrm{e}-12$ & -12.21171 & -11.96628 & -12.14659 \\
1 & $42.78135^{*}$ & $1.08 \mathrm{e}-17^{*}$ & $-23.62624^{*}$ & $-21.15367^{*}$ & $-23.23556^{*}$ \\
2 & 22.49615 & $4.43 \mathrm{e}-17$ & -22.83377 & -21.13407 & -22.11754 \\
\hline
\end{tabular}

Note: $*$ indicates lag order selected by the criterion

TABLE 4: Statistical results of the trace tests.

\begin{tabular}{|c|c|c|c|c|}
\hline Null hypothesis & Eigenvalue & Trace statistic & $5 \%$ critical values & $P$ value \\
\hline None $^{*}$ & 0.979054 & 141.3837 & 69.81889 & 0.0001 \\
\hline At most 1 & 0.587154 & 44.73895 & 47.85613 & 0.0953 \\
\hline At most 2 & 0.366389 & 22.62196 & 29.79707 & 0.2652 \\
\hline
\end{tabular}

TABLE 5: Statistical results of the maximum eigenvalue test.

\begin{tabular}{|c|c|c|c|c|}
\hline Null hypothesis & Eigenvalue & Max-Eigen statistic & $5 \%$ critical values & $P$ value \\
\hline None $^{*}$ & 0.979054 & 96.64470 & 33.87687 & 0.0001 \\
\hline At most 1 & 0.587154 & 22.11700 & 27.58434 & 0.2144 \\
\hline At most 2 & 0.366389 & 11.40800 & 21.13162 & 0.6066 \\
\hline
\end{tabular}

performed on the first difference intensely demonstrate that the first difference of each time series is stationary at the 5\% significance level; that is, these series are integrated of order first.

As for the cointegration test, variables are required to be integrated. The stationarity test result indicates that all variables are integrated of order first, which is reasonable to perform the cointegration test. There are two mainly used cointegration test methods: the Engle-Granger (E-G) test based on the regression residual applied to the bivariate and the Johansen-Juselius (JJ) test based on the regression coefficient for the multivariate. In our model, the JJ test method is applied.

Before the cointegration test, we should determine the optimal lag length, whose specific operation is expressed as follows: beginning from a larger lag order, the optimal lag length can be determined by comprehensive judgment through the LR value, FPE value, AIC value, SC value, and
HQ value. From Table 3, the test results demonstrate that the optimum lag length is one.

According to characteristics of the statistics, we select the linear tendency of the sequence, but the equation only has the test form of intercept. The statistical results of the trace test shown in Table 4 illustrate that null assumption of no cointegration relationships is rejected at the $5 \%$ significance level. As a result, there exists a cointegration relationship between variables. It can also obtain the same conclusion from the maximum eigenvalue test results in Table 5.

3.2. The Impulse Response Function Analysis. The stability of VAR model requires to be tested before the impulse response function analysis which is only suitable for the stable VAR model [38]. The model will be stable when estimated absolute values of all inverse characteristic roots of the characteristic equation are less than one; that is, inverse roots of $A R$ characteristic polynomial are inside the unit circle. 
TABLE 6: Findings from variance decomposition.

\begin{tabular}{|c|c|c|c|c|c|c|}
\hline Period & S.E. & $\operatorname{Ln}(\mathrm{EC})$ & $\operatorname{Ln}(\mathrm{P})$ & $\operatorname{Ln}(\mathrm{PGDP})$ & $\operatorname{Ln}(\mathrm{T})$ & $\operatorname{Ln}(\mathrm{URB})$ \\
\hline 1 & 0.064050 & 100.0000 & 0.000000 & 0.000000 & 0.000000 & 0.000000 \\
\hline 2 & 0.093137 & 74.33587 & 5.534857 & 8.018522 & 2.012121 & 10.09863 \\
\hline 3 & 0.107519 & 63.05512 & 6.506209 & 11.79173 & 2.052927 & 16.59401 \\
\hline 4 & 0.114523 & 55.41137 & 6.884434 & 11.87527 & 4.203496 & 21.62543 \\
\hline 5 & 0.118258 & 52.07434 & 7.156252 & 11.72533 & 4.369504 & 24.67458 \\
\hline 6 & 0.120550 & 49.20459 & 7.509135 & 11.91758 & 5.478080 & 25.89061 \\
\hline 7 & 0.122556 & 47.85810 & 7.998223 & 12.59354 & 5.520573 & 26.02956 \\
\hline 8 & 0.124997 & 46.66049 & 8.603650 & 13.76629 & 5.519488 & 25.45009 \\
\hline 9 & 0.128081 & 45.52651 & 9.262990 & 16.29813 & 4.498583 & 24.41379 \\
\hline 10 & 0.131648 & 44.46939 & 9.910890 & 17.96772 & 4.472165 & 23.17983 \\
\hline
\end{tabular}

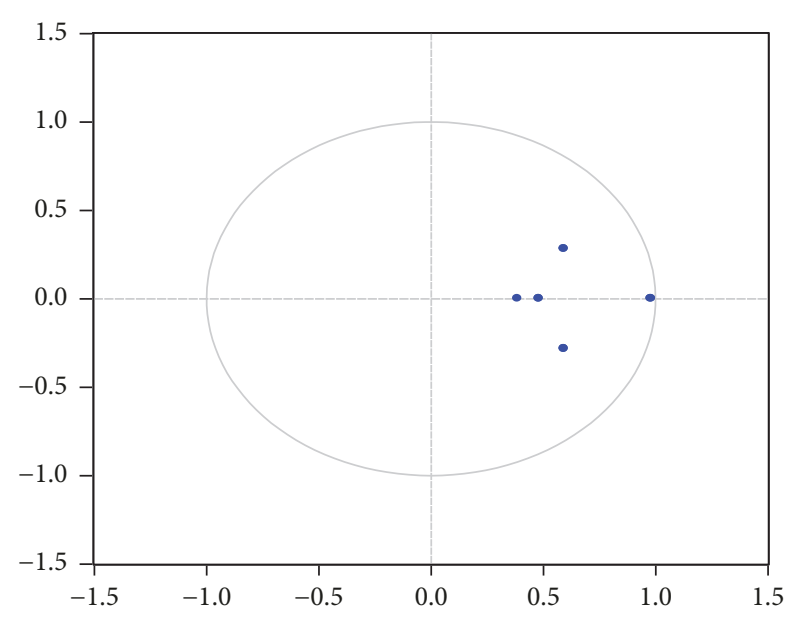

FIGURE 3: Finding from the stability test of VAR model.

From Figure 3, absolute values of all unit roots are inside the unit circle. Therefore, the VAR model meets the stability conditions so that impulse response function analysis can be explored.

From Figure 4, a shock to energy consumption positively effects itself, reaching the minimum in the fifth period, showing a U-shaped curve; a one standard deviation positive change to population positively affects energy consumption, climaxing in the second period, and this effect is stabilized after the seventh period; GDP responds positively to a one standard deviation positive change in energy consumption, and this response is relatively long-lived, reaching the maximum in the second period; a positive shock in technology negatively affects energy consumption after the third period, while this effect is very little previously; a positive change in the urbanization level exerts a positive effect on energy consumption, climaxing in the third period, showing an inverted U-shaped curve.

3.3. Variance Decomposition. Table 6 indicates that the shock to energy consumption account is $100 \%$ and $44.47 \%$ fluctuation in itself in the first quarter horizon and tenth quarter horizon, respectively, and it has been a downward trend from 1 to 10 quarters. Fluctuation in energy consumption from 0 to $9.91 \%$ can be explained by the innovation to population, showing a rising trend from the first period to the tenth period and can also be attributed to innovations in GDP ranging from 0 to $17.97 \%$, the innovation of which has been increasing over the ten quarters. The innovation to technology accounts for 0 and $4.47 \%$ fluctuation in energy consumption in the first horizon and tenth quarter horizon, respectively. The urbanization level can be attributed to innovations in energy consumption from 0 to $23.18 \%$.

3.4. Forecasting of $G M(1,1)$ Model. According to the Shanxibased energy consumption data, the prediction formula of GM $(1,1)$ model for the energy consumption can be expressed as

$$
\begin{aligned}
& \stackrel{\wedge}{X}^{(1)}(k+1)=79122.09 e^{-0.056 k}-74411.638 ; \\
& \stackrel{\wedge}{X}^{(0)}(k+1)=\stackrel{\wedge}{X}^{(1)}(k+1)-\stackrel{\wedge}{X}^{(0)}(k), \\
& k=1,2,3, \ldots
\end{aligned}
$$

The results of predicted values and actual ones from 1990 to 2015 are shown in Table 7. It can be calculated that the mean percentage error of $\mathrm{GM}(1,1)$ model is $7.264 \%$, the mean prediction accuracy is $92.736 \%$, the posterior variance ratio is 0.132 , and the $\mathrm{P}$ value of posterior variance test is 1.000 . In accordance with the optimum range of indicator values $C$ and $\mathrm{P}$, as aforementioned, the model produced approximately perfect estimates.

The energy consumption from 2016 to 2025 can be predicted by (19). As can be seen from Table 8, energy consumption of Shanxi Province is continually increasing in the next few years. By 2025, the energy consumption will increase to 31103.82 ten thousand tons of standard coal.

\section{Discussion}

The main drivers of energy consumption are based on the extended STIRPAT model. The results indicate the natural logarithm form of energy consumption, population, GDP, technology, and the urbanization level are all integrated of order first and subject to long-term equilibrium relationship. The VAR model can be built accordingly to probe into the dynamic connection between them. 

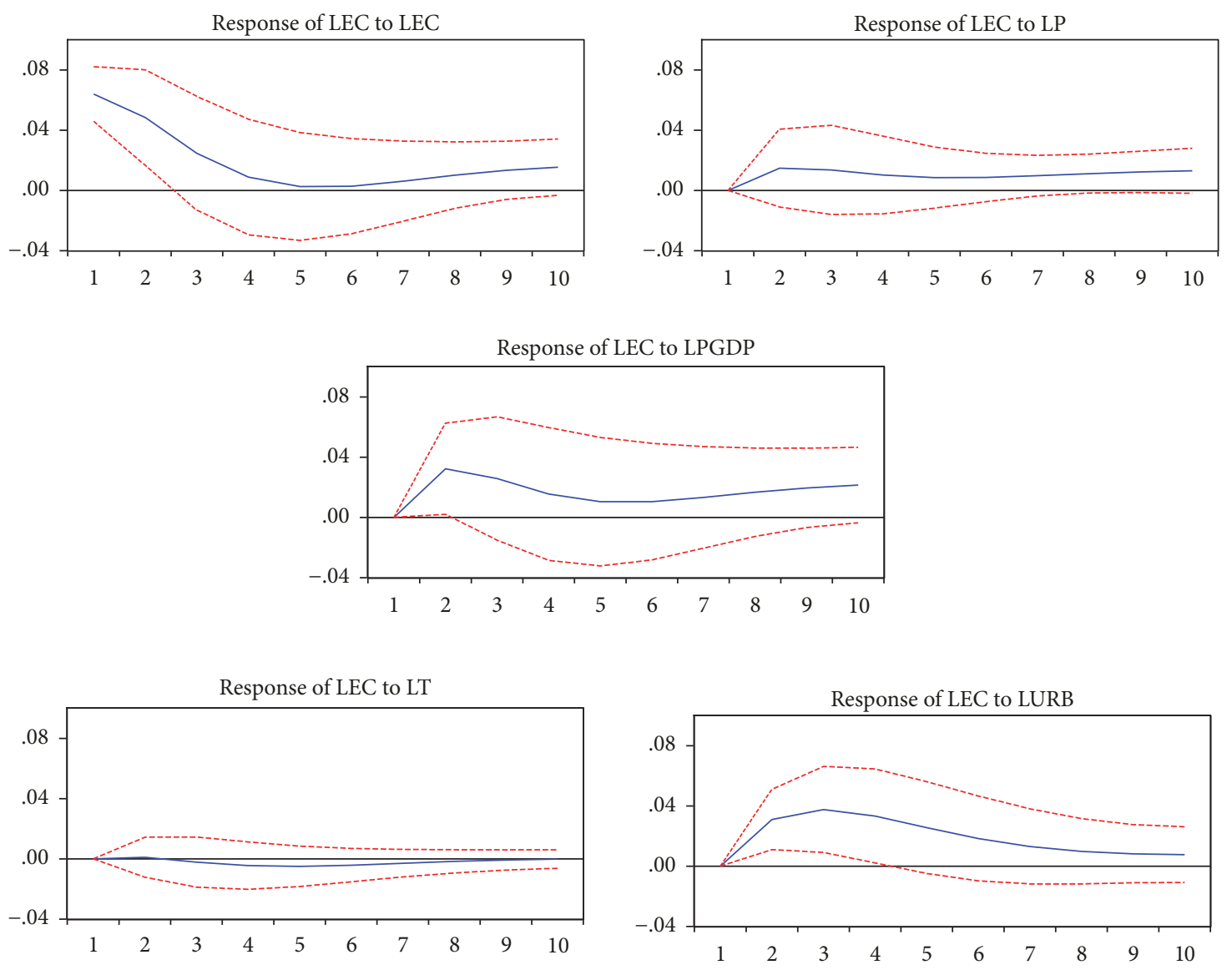

FIGURE 4: Findings from impulse response function.

Based on the impulse response analysis, a one standard deviation positive change to population positively affects energy consumption, showing that the population is a critical factor influencing the growth of energy consumption in Shanxi Province. The increase in population has augmented the demand for products and services, which has led to the growth of energy consumption demand.

GDP responds positively to a one standard deviation positive change in energy consumption, indicating that the economic growth has had a continuous effect on the growth of energy consumption in Shanxi Province. This is due to the reason that as the abundant coal resources in Shanxi Province, the local resource attributes determine that its longterm economic development will inevitably lead to the largescale development and utilization of energy.

A positive shock in technology negatively affects energy consumption after the third period, which can be attributed that technological progress cannot curb energy consumption in the short term. As it will take a long time that the new technology diffusion after research and development, technology can only play a role in reducing energy consumption after a period of accumulation. This result shows that, in the long term, technological progress is an effective means of reducing energy consumption.

A positive change in the urbanization level exerts a positive effect on energy consumption. The reason for this trend is that cities are the main body of energy consumption in China. Along with the advancement of urbanization, the demand for energy in urban buildings, transportation, and residential buildings is also increasing. As for Shanxi, the promotion of urbanization process mainly depends on the development of industry, which has intensified energy consumption accordingly. The response curve shows an inverted " $U$ " trend, indicating that there may exist an environmental Kuznets curve (EKC) between Shanxi's coal consumption and urbanization rate.

From the variance decomposition results, fluctuation in energy consumption from 0 to $9.91 \%$ can be explained by the innovation to population. Due to the continuous improvement of living standards, the demand for energy production and consumption will also increase as a result, and the impact of population effects on energy consumption will gradually enhance. The innovation to GDP for fluctuation in energy consumption continues to be increasing, which accounts for $17.97 \%$ in the long run, meaning that in medium and longterm the economy of Shanxi Province is in its extensive growing stage, and hence the resource-consuming economic growth is accompanied by an increase in energy consumption. The innovation to technology accounts for $4.47 \%$ fluctuation in the long run, indicating that the technology level contributes a small part to energy consumption, but raising the level of coal utilization and energy efficiency through 
TABLe 7: Prediction results from 1990 to 2015.

\begin{tabular}{|c|c|c|c|}
\hline Year & Actual values & Predicted values & Percentage predicted error \\
\hline 1991 & 4802.33 & 4583.83 & -4.55 \\
\hline 1992 & 5034.36 & 4849.38 & -3.67 \\
\hline 1993 & 5472.54 & 5130.33 & -6.25 \\
\hline 1994 & 5200.32 & 5427.54 & 4.37 \\
\hline 1995 & 6574.4 & 5741.98 & -12.66 \\
\hline 1996 & 6803.8 & 6074.63 & -10.72 \\
\hline 1997 & 6694.9 & 6426.56 & -4.01 \\
\hline 1998 & 6613.8 & 6798.87 & 2.80 \\
\hline 1999 & 5573.19 & 7192.76 & 29.06 \\
\hline 2000 & 5788.01 & 7609.46 & 31.47 \\
\hline 2001 & 6966.97 & 8050.30 & 15.55 \\
\hline 2002 & 8097.9 & 8516.68 & 5.17 \\
\hline 2003 & 9037.51 & 9010.09 & -0.30 \\
\hline 2004 & 9550.56 & 9532.07 & -0.19 \\
\hline 2005 & 10117.06 & 10084.30 & -0.32 \\
\hline 2006 & 11196.06 & 10668.52 & -4.71 \\
\hline 2007 & 12135.45 & 11286.58 & -6.99 \\
\hline 2008 & 12472.37 & 11940.46 & -4.26 \\
\hline 2009 & 12885.85 & 12632.21 & -1.97 \\
\hline 2010 & 13820.47 & 13364.04 & -3.30 \\
\hline 2011 & 14992.59 & 14138.27 & -5.70 \\
\hline 2012 & 15803.31 & 14957.35 & -5.35 \\
\hline 2013 & 16427.08 & 15823.88 & -3.67 \\
\hline 2014 & 16325.19 & 16740.61 & 2.54 \\
\hline 2015 & 15813.29 & 17710.46 & 12.00 \\
\hline
\end{tabular}

TABLE 8: Predicted values from 2016 to 2025.

\begin{tabular}{lc}
\hline Year & Predicted values \\
\hline 2016 & 18736.49 \\
2017 & 19821.96 \\
2018 & 20970.31 \\
2019 & 22185.20 \\
2020 & 23470.47 \\
2021 & 24830.20 \\
2022 & 26268.70 \\
2023 & 27790.54 \\
2024 & 29400.54 \\
2025 & 31103.82 \\
\hline
\end{tabular}

technological innovation is still a key factor in reducing energy consumption. A positive change in the urbanization level exerts a positive effect on energy consumption. This tendency mainly arises from the fact that the increase of urbanization rate has prominent contributions to medium and long-term energy consumption, because the burden of urban construction caused by agglomeration of rural people to cities has increased the use of fuel and cement and fossil energy consumption in daily life.

$\mathrm{GM}(1,1)$ model can be established to perfectly predict the energy consumption of Shanxi Province. According to the forecasting model, it is expected that the energy consumption of 2025 of Shanxi will reach 31103.82 ten thousand tons of standard coal. As a result, the government and relevant departments should effectively adopt reasonable policies to increase energy efficiency to avoid the crisis of energy exhaustion and realize the sustainable development.

\section{Conclusions and Policy Implications}

This paper explores dynamic relationship between the driving forces determined by the extended STIRPAT model and energy consumption in Shanxi Province through VAR model. We found clean energy represented by the natural gas accounted for a comparatively tiny proportion of the whole. It can be obtained from the impulse response analysis that a positive shock in population, GDP, and the urbanization level, respectively, exerts a positive effect on energy consumption, with all of those responses being relatively long-lived. And a positive change in technology negatively affects energy consumption in the long run. The variance decomposition results indicate that fluctuation in energy consumption can be respectively explained by the innovation to population, GDP, technology, and the urbanization level accounting for $9.91 \%, 17.97 \%, 4.47 \%$, and $23.18 \%$ in the long run. A large part of Shanxi's energy consumption growth can be explained by urbanization. Meanwhile, the forecast results of $\mathrm{GM}(1,1)$ 
model manifest that energy consumption in Shanxi Province generally has an increasing trend year by year.

These conclusions are illuminating to attract attention from local policy-makers. Specific implications are summarized as follows in aspects of energy structure, urbanization, and technology in order to ameliorate traditional coaldominating situation, earnestly implement the new Five Development Concept, and accomplish in-depth reform of resource-based economy and green sustainable development in Shanxi Province.

(1) Reform the Energy Structure and Propel Diversified Energy Consumption. Energy consumption of Shanxi is mainly dominated by the coal of primary energy source. Owing to its highpollution and nonrenewable properties, the overuse of coal should be slashed dramatically and green renewable energy deserves to be boosted actively. Thus, relevant departments ought to expedite the implementation of "coal to gas" projects for civil and industrial use and other key alternative areas.

As a province with abundant CBM resource, Shanxi has a well-distributed CBM field with good conditions, so that it would be helpful to increase the development and utilization of CBM resources. Meanwhile, with rich resources of solar and wind energy, it is necessary and beneficial to steadily and smoothly push forward the construction of photovoltaic bases and northern Shanxi wind power bases, which will be of great help for the amelioration of conventional coaldominating situation. The progress of other new energy sources should be strengthened persistently, with the development relationship between traditional and new energy sources being coordinated.

(2) Promote the Transformation of Resource-Based Cities and Clarify the Orientation of Urban Development Planning. The economic growth mode of Shanxi Province is mainly driven by resources consumption. The promotion of urbanization mainly relies on the development of industry, and the urbanization process has obviously aggravated energy consumption. Therefore, in the process of urbanization, according to the local energy supply and demand situation, the urbanization rate should be appropriately slowed down, and new urbanization should be promoted to guarantee green energy consumption, and the transformation of resourcebased cities should be promoted to target deep processing of resources, lengthen the coal industrial chain, and vigorously promote the novel coal chemical industry. At the same time, it is helpful to develop nonresource industries, establish high-quality cereals producing areas, vigorously develop the tourism industry, and promote the integration of culture and tourism.

Shanxi should correctly understand the development stage and characteristics of the city, define the future direction and path of the city, and give full play to cities' agglomeration and radiation potential. It is of great significance to strengthen the cooperation in division of labor among big cities and its surrounding small and medium-sized ones, promote the integration process of the urban agglomerations and the integration of tourism resources, and build Shanxi Merchant Culture tourism boutique line. The local government should continue to expand the implementation scope of the "Hundred Town Construction Projects" and explore more potential small towns to give them a certain policy inclination and form a group of specialized towns with special characteristics.

(3) Increase Environmental Investment and Enhance LowCarbon Technological Innovation. The environmental technology should be given top priority, playing its critical role in technological progress in the energy conservation and development of low-carbon economy. Financial subsidies for "coal-to-electricity" of rural power grid reform and optimization project should be augmented to actualize a virtuous circle of energy production system.

Priorities for technology aspect should be vigorously driving the comprehensive utilization of coal to stimulate key areas such as coking and coal chemical industry to implement technology transformation for cleaner production. In addition, it is vital to continuously enhance the capability of independent innovation of the coal industry, extending the value chain of coal resources and increasing added value of coal industry. Furthermore, the implementation of ultra-low emissions and energy-saving transformation for coal-fired units and clean coal power generation technology will improve the overall quality of coal industry in Shanxi Province.

\section{Data Availability}

The data used to support the findings of this study are available from the corresponding author upon request.

\section{Conflicts of Interest}

The authors confirm that the mentioned received funding in Acknowledgments did not lead to any conflicts of interest regarding the publication of this manuscript. And the authors declare there are no conflicts of interest in the manuscript.

\section{Acknowledgments}

This work was supported by the National Natural Science Foundation of China (no. 71471061) and the National Social Science Foundation of China (no. 15BGL145).

\section{References}

[1] W.-M. To and P. K. C. Lee, "Energy consumption and economic development in Hong Kong, China," Energies, vol. 10, no. 11, 2017.

[2] H. Yu, "The influential factors of China's regional energy intensity and its spatial linkages: 1988-2007," Energy Policy, vol. 45, pp. 583-593, 2012.

[3] W. Lu, C. Chen, M. Su, B. Chen, Y. Cai, and T. Xing, "Urban energy consumption and related carbon emission estimation: a study at the sector scale," Frontiers of Earth Science, vol. 7, no. 4, pp. 480-486, 2013.

[4] S. H. Deng, J. Zhang, F. Shen, H. Guo, Y.-W. Li, and H. Xiao, “The relationship between industry structure, household-number 
and energy consumption in China," Energy Sources, Part B: Economics, Planning, and Policy, vol. 9, no. 4, pp. 325-333, 2014.

[5] L. Meng and J. Sager, "Energy consumption and energy-related $\mathrm{CO} 2$ emissions from China's petrochemical industry based on an environmental input-output life cycle assessment," Energies, vol. 10, no. 10, p. 1585, 2017.

[6] H. R. Cui, T. Zhao, and H. J. Shi, "STIRPAT-based driving factor decomposition analysis of agricultural carbon emissions in Hebei China," Polish Journal of Environmental Studies, vol. 27, no. 4, p. 1449, 2018.

[7] Energy Statistics Division of National Bureau of Statistics of China, China Energy Statistical Yearbook of 2016, China Statistics Press, Beijing, China, 2017.

[8] X. L. Pan, "Thoughts on establishing ecological rehabilitation mechanism," Shanxi Finance and Tax, vol. 1, no. 1, 2016.

[9] U. Soytas and R. Sari, "Can China contribute more to the fight against global warming?” Journal of Policy Modeling, vol. 28, no. 8, pp. 837-846, 2006.

[10] A. Talha Yalta and H. Cakar, "Energy consumption and economic growth in China: a reconciliation," Energy Policy, vol. 41, pp. 666-675, 2012.

[11] M. Zhang and Y. Song, "Exploring influence factors governing the changes in China's final energy consumption under a new framework," Natural Hazards, vol. 78, no. 1, pp. 653-668, 2015.

[12] H. N. Li, H. L. Mu, and M. Zhang, "Analysis of Chinas energy consumption impact factors," Procedia Environmental Sciences, vol. 824, no. 11 (PART B), p. 824, 2011.

[13] H. Xie, G. Liu, Q. Liu, and P. Wang, "Analysis of spatial disparities and driving factors of energy consumption change in China based on spatial statistics," Sustainability, vol. 6, no. 4, pp. 22642280, 2014.

[14] H. T. Pao, H. C. Fu, and C. L. Tseng, "Forecasting of $\mathrm{CO}_{2}$ emissions, energy consumption and economic growth in China using an improved grey model," Energy, vol. 40, no. 1, pp. 400409, 2012.

[15] P.-P. Xiong, Y.-G. Dang, T.-X. Yao, and Z.-X. Wang, “Optimal modeling and forecasting of the energy consumption and production in China," Energy, vol. 77, pp. 623-634, 2014.

[16] X. Y. Wang, D. K. Luo, X. Zhao, and Z. Sun, "Estimates of energy consumption in China using a self-adaptive multi-verse optimizer-based support vector machine with rolling," Energy, vol. 152, no. 539, 2018.

[17] X. Wang and Z. Feng, "Energy consumption with sustainable development in developing country: a case in Jiangsu, China," Energy Policy, vol. 31, no. 15, pp. 1679-1684, 2003.

[18] J. Cai and Z. Jiang, "Changing of energy consumption patterns from rural households to urban households in China: an example from Shaanxi Province, China," Renewable \& Sustainable Energy Reviews, vol. 12, no. 6, pp. 1667-1680, 2008.

[19] L. Ren, W. Wang, J. Wang, and R. Liu, "Analysis of energy consumption and carbon emission during the urbanization of Shandong Province, China," Journal of Cleaner Production, vol. 103, pp. 534-541, 2015.

[20] C. Chong, L. Ma, Z. Li, W. Ni, and S. Song, "Logarithmic mean Divisia index (LMDI) decomposition of coal consumption in China based on the energy allocation diagram of coal flows," Energy, vol. 85, pp. 366-378, 2015.

[21] Y. Y. Feng, S. Q. Chen, and L. X. Zhang, "System dynamics modeling for urban energy consumption and $\mathrm{CO}_{2}$ emissions: a case study of Beijing, China," Ecological Modelling, vol. 252, no. 1, pp. 44-52, 2013.
[22] H. Zhang and M. L. Lahr, "China's energy consumption change from 1987 to 2007: a multi-regional structural decomposition analysis," Energy Policy, vol. 67, pp. 682-693, 2014.

[23] S. Wang, C. Fang, X. Guan, B. Pang, and H. Ma, "Urbanisation, energy consumption, and carbon dioxide emissions in China: a panel data analysis of China's provinces," Applied Energy, vol. 136, pp. 738-749, 2014.

[24] B. Z. Wang and J. Y. Huang, "Relationship between energy supply, energy consumption and economic growth: emprical study based on data on Shanxi Province from 1978 to 2008," Technology Economics, vol. 29, no. 2, p. 74, 2010.

[25] L. N. Xu, T. Zhao, J. S. Sun, and X. F. Yang, "Dynamic analysis between urbanization and energy consumption based on SVAR model in Shanxi province," Journal of Arid Land Resources and Environment, vol. 28, no. 9, 2014.

[26] W. Li and G. Yang, "Study on the sustainable development of energy consumption in shanxi province based on system dynamics," Resources Science, vol. 32, no. 10, 2010.

[27] M. J. Zhai, "Predict of Shanxi Provinces energy consumption based on ARIMA model," Mathematics in Practice and Theory, vol. 43, no. 7, p. 32, 2013.

[28] Z. X. Wang, "Multivariable GM(1, N) model with interaction effects," Control and Decision, vol. 32, no. 3, p. 515, 2017.

[29] C. A. Sims, "Macroeconomics and reality," Econometrica, vol. 48, no. 1, pp. 1-48, 1980.

[30] J. L. Deng, "Introduction to grey system theory," The Journal of Grey System, vol. 1, no. 1, pp. 1-24, 1989.

[31] P. R. Ehrlich and J. P. Holdren, "Impact of population growth," Science, vol. 171, no. 3977, pp. 1212-1217, 1971.

[32] T. Dietz and E. A. Rosa, "Rethinking the environmental impacts of population, Affluence and technology," Human Ecology Review, vol. 1, no. 2, p. 277, 1994.

[33] P. T. Soulé and J. L. DeHart, "Assessing IPAT using productionand consumption-based measures of I," Social Science Quarterly, vol. 79, no. 4, pp. 754-765, 1998.

[34] Shanxi Provincial Development and Reform Commission, "Outline of the 13th Five-Year Plan of Shanxi Province for Economic and Social Development," http://www.sxdrc.gov.cn/ xxlm/fzgh2/zhdt/201605/t20160503_174212.htm, 2016.

[35] H. J. Shan, "Reestimating the capital stock of China:1952-2006," The Journal of Quantitative \& Technical Economics, vol. 10, p. 17, 2008.

[36] P. Kennedy, A Guide to Econometrics, vol. 2, MIT press, 2003.

[37] G. W. Schwert, "Effects of model specification on tests for unit roots in macroeconomic data," Journal of Monetary Economics, vol. 20, no. 1, pp. 73-103, 1987.

[38] X. T. Zhang, Econometrics Software Eviews User Guide, Nankai University Press, 2004. 


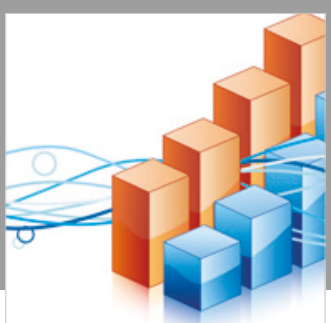

Advances in

Operations Research

\section{-n-m}
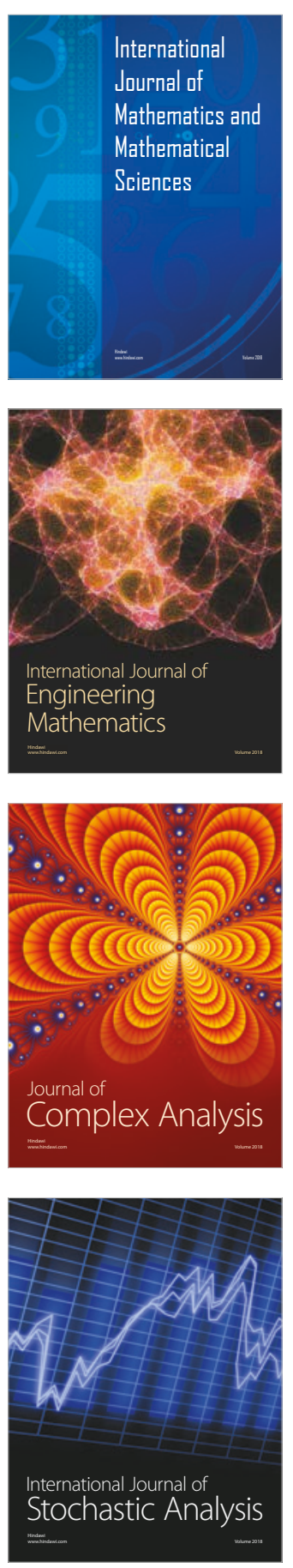
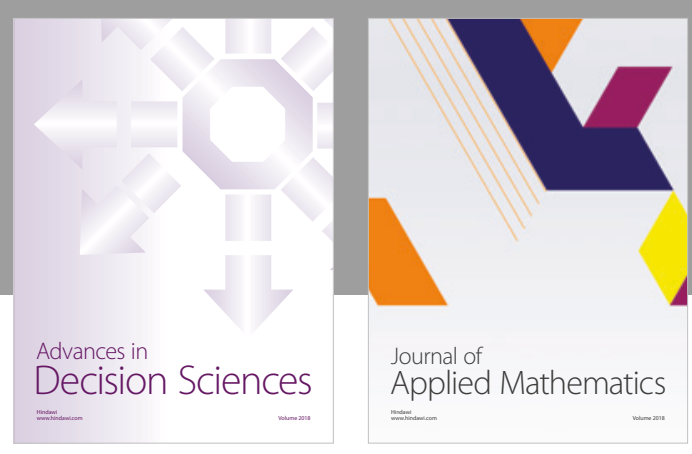

Journal of

Applied Mathematics
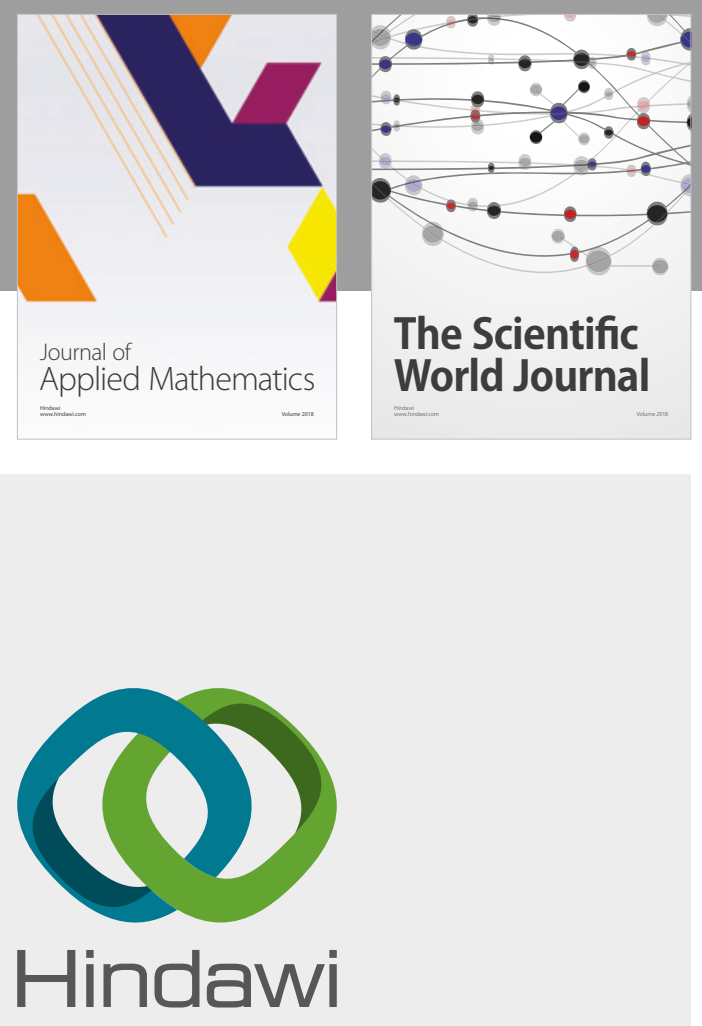

Submit your manuscripts at

www.hindawi.com

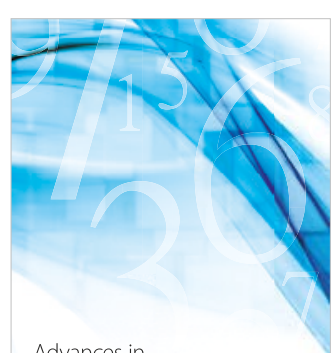

Advances in
Numerical Analysis
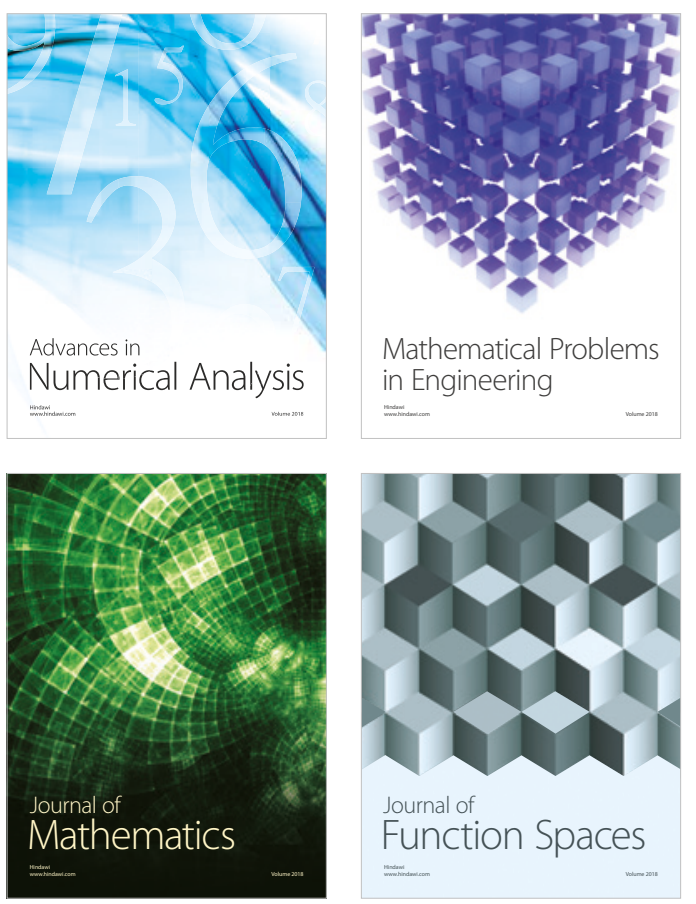

Mathematical Problems in Engineering

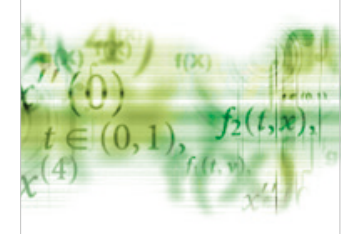

International Journal of

Differential Equations

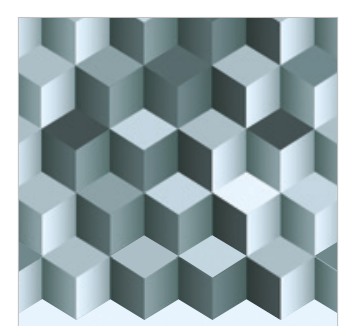

Journal of

Function Spaces

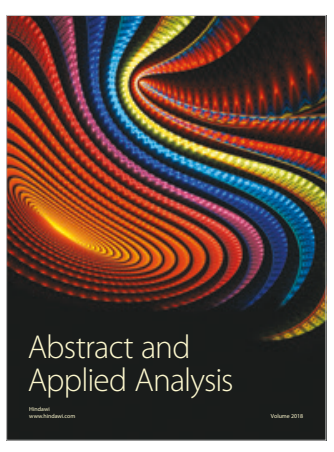

The Scientific

World Journal

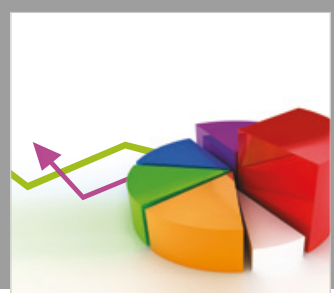

Journal of

Probability and Statistics
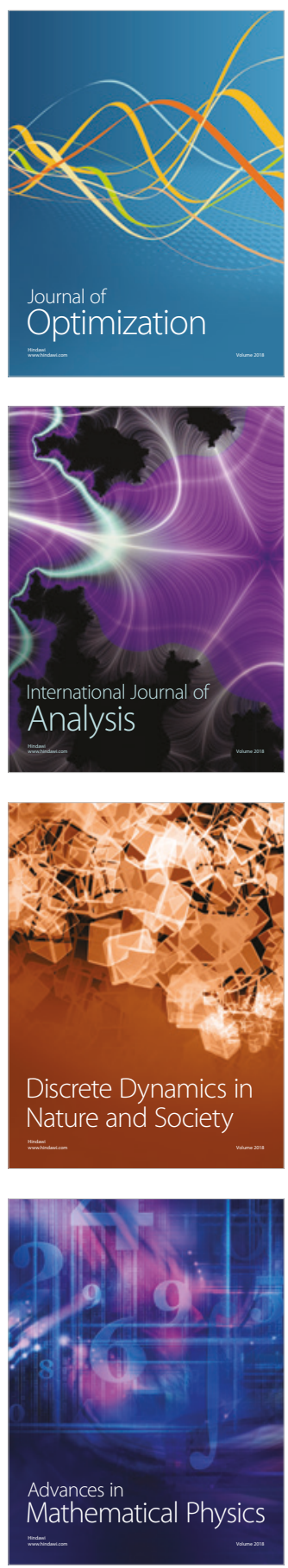